\title{
Dynamics of Active Mode Locking in Broad-Band Continuous Wave Lasers
}

\author{
K. P. J. Reddy and Jim A. Tatum
}

\begin{abstract}
Theoretical analysis for the dynamics of mode locking in an AM mode-locked broad-band tunable solid-state laser is presented. The analysis is applied to study the pulse evolution inside the $\mathrm{AM}$ mode-locked $\mathrm{Ti}: \mathrm{Al}_{2} \mathrm{O}_{3}$ laser. It is shown that the pulsewidth reaches a steady-state value after about 77500 round-trips inside the laser cavity which corresponds to $258 \mu \mathrm{s}$. An expression for the threshold condition for the mode-locking process is presented. The analysis yields the design parameters required for building an AM mode-locked laser.
\end{abstract}

\section{INTRODUCTION}

$\mathrm{U}$ LTRASHORT light pulses of picosecond and subpicosecond duration are routinely used for scientific, medical, and industrial applications. Commonly, these pulses are produced by the mode-locked dye lasers which are capable of generating pulses broadly tunable in the near-UV to near-IR region. However, the dye lasers have a major drawback due to the photodegradation of the dye solution. Recently, broadly tunable solid-state lasers have been found to provide an attractive alternative to dye lasers in the near infrared region [1].

Mode locking of tunable solid-state lasers has been achieved using active [2], passive [3], self- [4], synchronous [5], additive-pulse mode locking [6], and resonant passive mode-locking [7] techniques. Of all the tunable solid-state lasers $\mathrm{Ti}$ : sapphire has been extensively investigated both experimentally and theoretically [8]. Modelocked pulses of 11 fs duration have been produced recently in an intracavity dispersion compensated self-modelocked $\mathrm{Ti}$ : sapphire laser [9]. However, there are many scientific and engineering applications which need tunable picosecond pulses with high reliability. Active mode locking is the best suited technique for producing these pulses from broad-band solid-state lasers. This method is free from the stability and reproducibility problems faced by the other methods of mode locking. Hence efforts are being made to understand the characteristics of active mode locking of broadly tunable lasers. Recently, Darrow and Jain [8] published detailed analysis of the actively mode-locked broad-band lasers and their theoretical re-

Manuscript received June 22, 1992; revised November 9, 1992. The work of K. P. J. Reddy was supported by an Indo-US Fellowship for science and technology from USAID and DST (India).

K. P. J. Reddy is with the Department of Aerospace Engineering, Indian Institute of Science, Bangalore, 560012, India.

J. A. Tatum is with the Erik Jonsson School of Engineering and Computer Science, The University of Texas at Dallas, Richardson, TX 75080.

IEEE Log Number 9207832. sults compared well with the experimental results of $\mathrm{Ti}: \mathrm{Al}_{2} \mathrm{O}_{3}$ laser. In their paper the analysis is based on the theoretical model developed earlier by Kuizenga and Siegman $[10]$ for active mode locking of a solid-state laser. In this model a steady-state Gaussian pulse is assumed to circulate in the laser resonator and after traversing the intracavity elements and after experiencing the cavity loss, the pulse that returns to the starting point is assumed to stay unchanged. The extension of this model in [8] is achieved for broadly tunable solid-state lasers by including a bandwidth tuning element inside the laser cavity and accounting for the effect of dispersion in the intracavity optical components on the mode-locked laser pulse. However, the analysis concentrates on the steady-state characteristics and has paid no attention to the pulse forming dynamics. Studies of mode-locking dynamics have been reported recently for other techniques of mode locking in $\mathrm{Ti}$ :sapphire laser where it has been shown that complete mode locking is achieved after $200 \mu \mathrm{s}$ in selfstarting additive-pulse mode locking [12], millisecond, and $230 \mu$ s in passive mode locking by HITCI [12] and DDI [13], respectively.

In this paper we report the theoretical study of the dynamics of active mode locking of broad-band CW solidstate lasers. The primary interest in this paper is focused on the temporal evolution of the pulsewidth and the pulsewidth-bandwidth product of the mode-locked pulse inside the laser cavity. The importance of the analysis lies in the fact that first it helps in determining the time interval required to complete the mode-locking process of the time interval required to recover if the mode-locked laser is perturbed. Second, the analysis results in the determination of the modulation index required to achieve mode locking, which is important for designing the modulator. The approach used in the analysis is essentially an extension of the self-consistent theory of Kuizenga and Siegman incorporating the modifications for the broad-band laser systems suggested in [8]. Although we have considered here only the case of AM mode locking with zero detuning, the analysis can be easily extended for the finite detuning and FM mode locking.

\section{ANALYSIS}

The analysis proceeds by assuming a Gaussian pulse to circulate in the laser resonator. Necessary approximations are made to the transmission functions of the intracavity 
elements such as laser gain medium, bandwidth limiting element, and the acoustooptic modulator to keep the pulse Gaussian. The effect of each intracavity element on the parameter $\gamma$ defining the pulse parameters is separately calculated. Unlike in the steady-state theory, in this analysis the total change in the Gaussian pulse shape parameter is equated to differential increment $(\Delta \gamma)$ in the shape parameter. This leads to a differential equation in terms of the parameter $\gamma$. The solutions of this differential equation are then used to obtain the temporal variation of the pulsewidth and pulsewidth-bandwidth product of the Gaussian pulse. Finally, the fundamental differential equation governing the mode-locking process is examined to determine the threshold value of the modulation index necessary to achieve mode locking.

The electric field of the circulating Gaussian pulse inside the laser cavity is assumed in the form, $E(t)=\left(E_{0} / 2\right)$ $\exp \left(-\gamma t^{2}\right) \exp \left(j \omega_{p} t\right)$ where $\omega_{p}$ is the center frequency and $\gamma=\alpha-j \beta$ is the shape factor in which $\alpha$ and $\beta$ are related to the pulsewidth and the pulsewidth-bandwidth product through the equations $\tau_{p}=(2 \ln 2 / \alpha)^{1 / 2}$ and $\tau_{p}$. $\Delta f_{p}=(2 \ln 2 / \pi)\left(1+(\beta / \alpha)^{2}\right)^{1 / 2}$, respectively. The change in $\gamma$ due to the passage of the pulse through the homogeneously broadened broad-band laser medium with the gain $g(\omega)=G \exp \left[-(2 j g / \Delta \omega)\left(\omega-\omega_{a}\right)-\right.$ $\left.\left(4 g / \Delta \omega^{2}\right)\left(\omega-\omega_{a}\right)^{2}\right]$ where, $G=e^{g}, g$ is the saturated round-trip amplitude gain, $\omega_{a}$ is the atomic line center, and $\Delta \omega$ is the atomic linewidth, is given by

$$
\Delta \gamma_{A}=-16 g \gamma^{2} / \Delta \omega^{2} \text {. }
$$

Here we have assumed that $\omega_{a}=\omega_{p}$ (no detuning).

The transmission function through the host of the gain medium, focusing optical cavity components, cavity bandwidth limiting elements, and the medium of the modulator is assumed in the form [8],

$$
\begin{aligned}
\tau_{\text {cavity }}(\omega)= & \exp \left[-j\left(\left.P\right|_{\lambda=\lambda_{a}}\right) \omega_{a} / c\right] \\
& \cdot \exp \left\{(j / c) \lambda_{a}^{2}\left[d /\left.d \lambda(P / \lambda)\right|_{\lambda=\lambda_{a}}\right]\left(\omega-\omega_{a}\right)\right\} \\
& \cdot \exp \left[\left(-4 / \Delta \omega_{t}^{2}-j D / \pi^{2}\right)\left(\omega-\omega_{a}\right)^{2}\right]
\end{aligned}
$$

where $P$ is the round-trip optical path length, $c$ is the speed of light in vacuum, $\lambda_{a}$ is the wavelength of light at the gain peak, $\Delta \omega_{t}$ is the bandwidth of the tuning element, and $D=\left(\lambda_{a}^{3} / 8 c^{2}\right)\left(d^{2} P /\left.d \lambda^{2}\right|_{\lambda=\lambda_{a}}\right)$ represents the roundtrip cavity group velocity dispersion. The change introduced by the transmission function (2) in $\gamma$ is given by

$$
\Delta \gamma_{\text {cavity }}=-4 \gamma^{2}\left(4 / \Delta \omega_{t}^{2}+j D / \pi^{2}\right) .
$$

Assuming the round-trip transmission of the amplitude modulator in the form $a(t)=\exp \left(-\theta_{m}^{2} \omega_{m}^{2} t^{2}\right)$ where $\theta_{m}$ is the peak round-trip depth of amplitude modulation and $\omega_{m}$ is the modulation frequency, the change in $\gamma$ due to the passage through the modulator is given by

$$
\Delta \gamma_{A M}=\theta_{m}^{2} \omega_{m}^{2}
$$

The net change in $\gamma$ during one round-trip time is given by the summation of (1), (3), and (4). Thus

$$
\begin{aligned}
\Delta \gamma / \Delta t= & {\left[\theta_{m}^{2} \omega_{m}^{2}-16\left(g / \Delta \omega^{2}+1 / \Delta \omega_{t}^{2}\right.\right.} \\
& \left.\left.+j D / 4 \pi^{2}\right) \gamma^{2}\right](c / 2 L)
\end{aligned}
$$

where $\Delta t=2 L / c$ is the round-trip traversal time for the pulse inside the laser cavity of length $L$.

Assuming the changes in $\gamma$ during one round-trip time interval are small, the ratio $\Delta \gamma / \Delta t$ may be replaced by the continuous time derivative $d \gamma / d t$. Therefore, the above equation is rewritten as

$$
\begin{aligned}
d \gamma / d t= & \left(\theta_{m}^{2} \omega_{m}^{2}\right)(c / 2 L)-16 \gamma^{2}\left(g / \Delta \omega^{2}\right. \\
& \left.+1 / \Delta \omega_{t}^{2}+j D / 4 \pi^{2}\right)(c / 2 L) .
\end{aligned}
$$

Solving this equation by the method of separation of variables, we get the transient solution in the form [14], [15],

$$
\gamma=\gamma_{0} \tanh \left[(\sigma+j \Omega)\left(t+t_{0}\right)(c / 2 L)\right]
$$

where $t_{0}$ is a constant of integration and

$$
\begin{gathered}
\gamma_{0}=-\left[\theta_{m}^{2} \omega_{m}^{2} / 16\left(g / \Delta \omega^{2}+1 / \Delta \omega_{t}^{2}+j D / 4 \pi^{2}\right)\right]^{1 / 2} \\
\sigma=\operatorname{Re}\left[\theta_{m}^{2} \omega_{m}^{2} 16\left(g / \Delta \omega^{2}+1 / \Delta \omega_{t}^{2}+j D / 4 \pi^{2}\right)\right]^{1 / 2}
\end{gathered}
$$

and

$$
\Omega=\operatorname{Im}\left[\theta_{m}^{2} \omega_{m}^{2} 16\left(g / \Delta \omega^{2}+1 / \Delta \omega_{t}^{2}+j D / 4 \pi^{2}\right)\right]^{1 / 2} .
$$

At steady state, (6) gives

$$
\left.\gamma=4 \theta_{m} \omega_{m}\left(g / \Delta \omega^{2}+1 / \Delta \omega_{t}^{2}+j D / 4 \pi^{2}\right)\right]^{-1 / 2}
$$

from which, assuming $\left(g / \Delta \omega^{2}\right) \ll\left(1 / \Delta \omega_{f}^{2}\right)$ and/or $\left(D / 4 \pi^{2}\right)$, we can show that the steady-state pulsewidth and pulsewidth-bandwidth products are given by

$$
\begin{aligned}
\tau_{p s}= & \left(2^{3 / 2} \ln 2 / \theta_{m} f_{m} \pi^{2}\right)^{1 / 2} \cdot\left[\left(1 / \Delta f_{t}^{2}\right)^{2}+D^{2}\right]^{1 / 4} \\
& \cdot\left\{\left[\left(1 / \Delta f_{t}^{2}\right)^{2}+D^{2}\right]^{1 / 2}+\left(1 / \Delta f_{t}^{2}\right)\right\}^{-1 / 4}
\end{aligned}
$$

and

$$
\begin{aligned}
\tau_{p s} \Delta f_{p s}= & \left(2^{3 / 2} \ln 2 / \pi\right)\left\{1+\left(1 / \Delta f_{t}^{2}\right)\left[\left(1 / \Delta f_{t}^{2}\right)^{2}\right.\right. \\
& \left.\left.+D^{2}\right]^{-1 / 2}\right\}^{-1 / 2}
\end{aligned}
$$

These steady-state equations for pulsewidth and pulsewidth-bandwidth product are identical to the corresponding equations derived using steady-state analysis in [8].

Separating real and imaginary parts from (7) we can show that

$$
\begin{aligned}
\alpha= & \left(\theta_{m} \omega_{m} / 2^{5 / 2} Z^{1 / 2}\right)\left[X^{1 / 2} \sinh (2 \sigma M)\right. \\
& \left.+Y^{1 / 2} \sinh (2 \Omega M)\right] \\
& \cdot[\cosh (2 \sigma M)+\cosh (2 \Omega M)]^{-1}
\end{aligned}
$$




$$
\begin{aligned}
\beta= & \left(\theta_{m} \omega_{m} / 2^{5 / 2} Z^{1 / 2}\right)\left[Y^{1 / 2} \sinh (2 \sigma M)\right. \\
& \left.-X^{1 / 2} \sinh (2 \Omega M)\right] \\
& \cdot[\cosh (2 \sigma M)+\cosh (2 \Omega M)]^{-1}
\end{aligned}
$$

where

$$
\begin{aligned}
Z & =\left(g / \Delta \omega^{2}+1 / \Delta \omega_{t}^{2}\right)^{2}+\left(D / 4 \pi^{2}\right)^{2} \\
X & =Z^{1 / 2}+\left(g / \Delta \omega^{2}+1 / \Delta \omega_{t}^{2}\right) \\
M & =(c / 2 L)\left(t+t_{0}\right) .
\end{aligned}
$$

Here, $M$ is the number of round-trips the mode-locked pulse makes inside the laser cavity. Using (12a) and (12b) the time dependence of pulsewidth and pulsewidth-bandwidth product are obtained as

$$
\begin{aligned}
\tau_{p}= & \tau_{p s}\left[\sinh (2 \sigma M)+(Y / X)^{1 / 2} \sinh (2 \Omega M)\right]^{-1 / 2} \\
& \cdot[\cosh (2 \sigma M)+\cosh (2 \Omega M)]^{1 / 2} \\
\tau_{p} \Delta f_{p}= & \left(\tau_{p s} \Delta f_{p s}\right)[\sinh (2 \sigma M) \\
& \left.+(Y / X)^{1 / 2} \sinh (2 \Omega M)\right]^{-1} \\
& \cdot\left[\sinh ^{2}(2 \sigma M)+\sinh ^{2}(2 \Omega M)\right]^{1 / 2}
\end{aligned}
$$

Equations (13) and (14) describe the dynamics of pulse evolution inside the mode-locked broad-band tunable solid-state lasers starting from the initial continuous wave (CW) operation. It is important to note that although we have considered only the case of AM mode locking with zero detuning the frequency chirp $\beta$ which contributes to the total bandwidth is not zero. The finite value of $\beta$ is due to the group velocity dispersion effects of the material media inside the laser cavity.

\section{Results AND Discussion}

The equations derived in the preceding section are applied to study the dynamics of AM mode-locking process in $\mathrm{Ti}$ : sapphire laser. The parameters considered are the saturated gain $g=0.18$, the gain bandwidth $\Delta f=197.2$ $\mathrm{THz}$, the tuning element bandwidth $\Delta f_{t}=56.9,13.8$, and $3.45 \mathrm{THz}$ corresponding to one, two and three plate birefringent filters, respectively, the modulation depth $\theta_{m}=$ 0.55 , the modulation frequency $f_{m}=160 \mathrm{MHz}$, and the laser cavity length $L=0.5 \mathrm{~m}$. The group velocity dispersion term is taken in the form $D=[62.0+(18.3$ $\left.\left.\mathrm{THz} / \Delta f_{t}\right)\right] \times 10^{-28} \mathrm{~s}^{2}[8]$.

The steady-state values of the pulsewidth and the pulsewidth-bandwidth products are computed from (10) and (11), respectively, while the time evolution of these variables are computed from (13) and (14) and the results are given in graphical form in Figs. 1 and 2. The mode-locking process reaches steady state when the transient values of pulsewidth and pulsewidth-bandwidth product approach the corresponding values predicted by the steadystate equations (10) and (11), respectively. Thus it is seen from Fig. 1, that starting from a very large initial value the pulsewidth approaches the steady state value after the mode-locked pulse makes very large number of round-

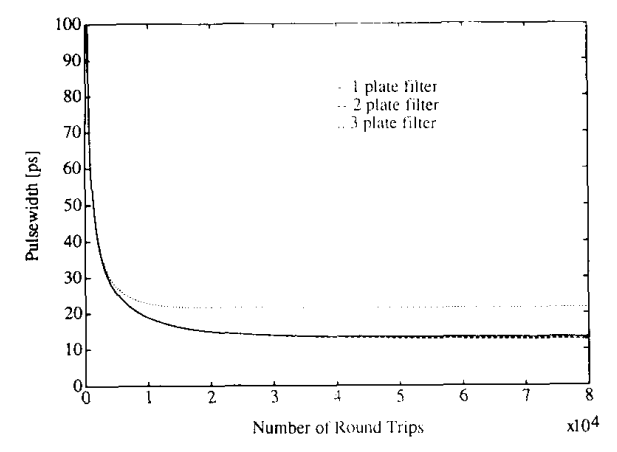

Fig. 1. Variation of the mode-locked pulsewidth as a function of number of round-trips inside the laser cavity for three different tuning elements.

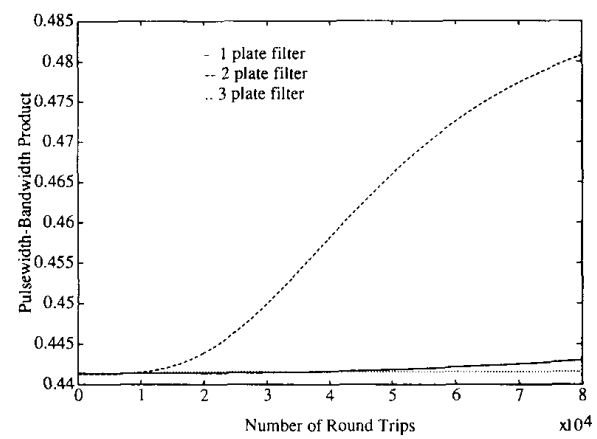

Fig. 2. Variation of the pulsewidth-bandwidth product as a function of number of round-trips inside the laser cavity for three different tuning elements.

trips inside the laser cavity. Therefore, the variation of pulse parameters in a single round-trip traversal through the intracavity elements is very low. Hence the transformation of the finite difference equation (5) into a differential form (6) is justified. Of the three tuning elements the three-plate filter produces the broadest steady-state pulses. Evolution of pulsewidth-bandwidth product shown in Fig. 2 indicates a largest value for the two plate filter. For the remaining two tuning elements this product remains constant and almost equal to an ideal value of 0.440 [10] for the AM case. Larger value of the pulsewidth bandwidth product is due to a finite value of $\beta$ which grows from zero with the increase of the number of roundtrips inside the cavity, as shown Fig. 3. The presence of frequency chirp indicated by $\beta \neq 0$ is due to the inclusion of dispersion effects in the analysis. Thus if $D=0$ then $\beta=0$ from $(12 b)$. It is important to note that due to the presence of frequency chirp the pulses from the AM modelocked $\mathrm{Ti}$ : sapphire laser with a two-plate tuning element could be further shortened by using pulse compression techniques such as a grating pair.

Comparing the pulsewidth evolution shown in Fig. 1 with the corresponding plot given in [13], it is seen that the oscillatory behavior noticed in the pulsewidth evolution in a passively mode-locked $\mathrm{Ti}$ : sapphire/DDI laser is not noticed in the present case. The oscillatory behavior in the passively mode-locked laser is due to the compe- 


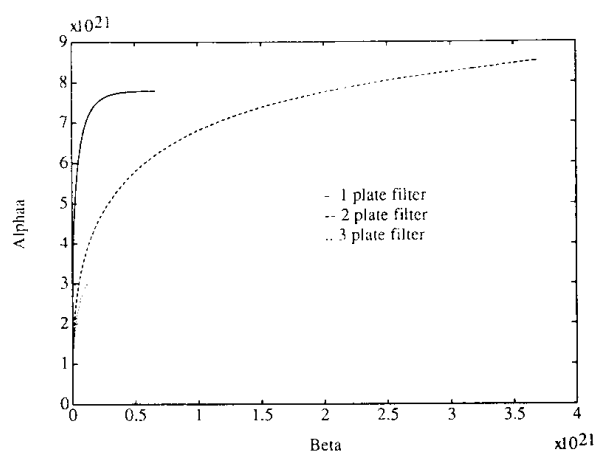

Fig. 3. Pulsewidth parameter $\alpha$ and frequency chirp parameter $\beta$ for the mode-locked laser with three different tuning elements.

tition between the pulse shortening and broadening forces in the cavity which occurs because of the statistical nature of the pulse evolution in this system. Since the modelocking process in active mode locking is free from fluctuations, the oscillatory behavior in pulse evolution is not noticed.

The dependence of mode-locked pulsewidth on the modulation depth is investigated and the steady-state values for various values of $\theta_{m}$ are plotted in Fig. 4. The curves shown here again indicate the broader pulses generated by three-plate tuning element at larger values of $\theta_{m}$. However, for lower values of $\theta_{m}$ all the three tuning elements produce identical values for the steady-state pulsewidth. Also it is noted that the dependence of pulsewidth on the modulation depth is very sensitive for lower values of $\theta_{m}$.

To estimate the exact time for pulse evolution it is necessary to evaluate the constant of integration $t_{0}$ in (7). With the knowledge of the initial conditions of the laser system $t_{0}$ could be estimated. However, it is difficult to estimate the initial values in a practical system. An alternative way is to estimate $t_{0}$ by estimating the initial value $\gamma_{m}$ of the parameter $\gamma$. This is achieved as follows. With the knowledge of average number of modes $N$ oscillating inside the laser cavity, and the mode separation frequency $f_{c}$, the initial value of the pulsewidth could be estimated using the relationship $\tau_{m}=1 / N f_{c}$. Since the average number of modes does not change, the number of modes can be experimentally measured using a scanning Fabry-Perot etalon. As $\beta=0$ at the beginning of the mode locking, as shown in Fig. 3, the initial value of $\gamma$ could be estimated as $\gamma_{m}=\alpha_{m}=2 \ln 2 / \tau_{m}^{2}$. For a typical mode-locked $\mathrm{Ti}$ : sapphire laser $N=25$ and $f_{c}=300 \mathrm{MHz}$, which yield $\tau_{m}=133.33 \mathrm{ps}$. Assuming that at $t=0, \tau_{p}=\tau_{m}(13)$ yields $\left(t_{0} c / 2 L\right)=2500$. The exact number of round-trips required to reach steady state is computed by subtracting this value from the number of round-trips at steady state shown in Fig. 1. Thus we see that the mode-locked pulse makes about 77500 round-trips inside an AM mode-locked $\mathrm{Ti}$ : sapphire laser to reach steady state. This corresponds to the pulse evolution time of $258.33 \mu \mathrm{s}$. This time is

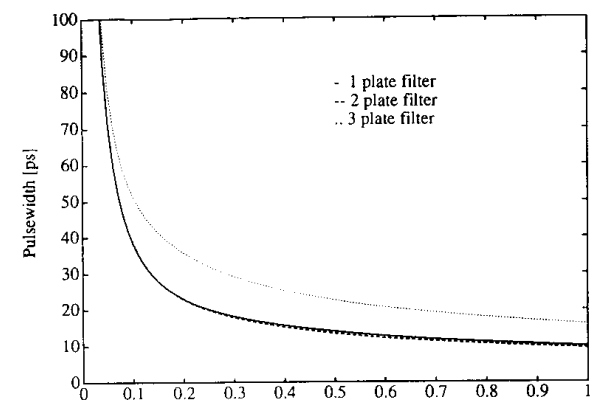

Modulation Depth

Fig. 4. Steady-state mode-locked pulsewidth variation as a function of the modulation depth of the AM modulator.

slightly higher than the recently measured value of $230 \mu \mathrm{s}$ in the $\mathrm{Ti}$ : sapphire/DDI passively mode-locked laser [13].

An estimation of the threshold condition for the mode locking could be obtained by examining the differential equation (6). It shows that the action of the AM modulator is to increase $\gamma$ (reduce $\tau_{p}$ ) and that of the laser medium and the tuning element is to decrease $\gamma$ (increase $\tau_{p}$ ). A steady state is attained when these two effects are balanced. For the estimated value of $\gamma_{m}$, a measure of modelocking threshold could be obtained from the equation

$$
\theta_{m}^{2}>\left(\gamma_{m}^{2} / \pi^{4} f_{m}^{2}\right)\left(g / \Delta f^{2}+1 / \Delta f_{t}^{2}+j D / 4 \pi^{2}\right)
$$

Since the effect of the modulator is real, the imaginary part could be neglected and the threshold value of $\theta_{m}$ could be estimated from

$$
\theta_{m}^{2} \sim(2 \ln 2)^{2}\left(\pi^{4} \tau_{m}^{2} f_{m}^{2}\right)^{-1}\left(g / \Delta f^{2}+1 / \Delta f_{\imath}^{2}\right)
$$

For the AM mode-locked $\mathrm{Ti}$ : sapphire laser considered here, this equation yields $\theta_{m}=3.85 \times 10^{-3}$. This value signifies the minimum value of the modulation depth required to achieve mode locking. To ensure mode locking and allow for small detuning effects, we can use a value $\theta_{m}=0.04$. With this value for the modulation depth the analysis predicts a steady-state pulsewidth of $46.89 \mathrm{ps}$.

The error introduced in the above calculations due to the error in the estimation of initial values is negligible. For example even assuming an infinite pulsewidth $\left(t_{0}=\right.$ 0 ) would introduce an error less than $3 \%$ because of the large number of round-trips the pulse needs to make inside the laser cavity before reaching the steady state. Thus the error introduced by the indeterminacy of the initial pulsewidth does not seriously affect the accuracy of the estimation of the pulse evolution time interval. Likewise the error introduced in measuring the number of oscillating modes which results in the initial pulsewidth estimate will also introduce an error less than $3 \%$ in the estimation of the threshold value of the modulation depth. Since the mode-locking threshold is not a well-defined quantity, this error is within the acceptable limit. 


\section{CONCLUSION}

The dynamics of pulse formation in AM mode-locked broadly tunable $\mathrm{CW}$ solid-state lasers has been investigated. The analysis is presented for zero detuning, but it could be easily extended for finite detuning and FM modelocking process. It is shown that the pulse makes about $7.75 \times 10^{4}$ round-trips inside the $\mathrm{AM}$ mode-locked Ti:sapphire laser before reaching the steady state. Thus the system reaches steady state after about $258 \mu$ s which is comparable to that of the passively mode-locked Ti : sapphire laser. An estimate of the threshold condition for the mode locking is also presented. For AM modelocked $\mathrm{Ti}$ : sapphire laser the threshold value of modulation depth to achieve mode locking is found to be equal to 0.04 . The error in the calculation of the above quantities is found to be less than acceptable limits.

The analysis thus helps in determining the parameters such as pulsewidth, the minimum modulation index required for mode locking and the time required for reaching the steady state, which characterize the laser. These parameters aid the design of the laser system.

\section{ACKNOWLEDGMENT}

We would like to thank D. L. MacFarlane for discussions.

\section{REFERENCES}

[1] F. J. Duarte and L. W. Hillman, Ed., Dye laser principles with applications. Boston, MA: Academic, 1990

[2] B. C. Johnson, P. F. Moulton, and A. Mooradian, "Mode-locked operation of $\mathrm{Co}: \mathrm{MgF} 2$ and $\mathrm{Ni}: \mathrm{MgF} 2$ lasers," Opt. Lett., vol. 10 , pp. 116-118, 1984; J. F. Pinto, C. P. Yakymyshyn, and C. R. Pollock, "Acousto-optic mode-locked soliton laser," Opt. Lett., vol. 13, pp. 383-385, 1988; J. T. Darrow, R. K. Jain, and M. Poelker, "Active and synchronous mode-locking of a room temperature $\mathrm{CW}$ titanium sapphire laser," in Conf. Dig., LEOS, Wash., DC: OSA, 1988, paper ME1

[3] N. Sakura. Y. Ishida, H. Nakano, and Y. Yamamoto, "CW passive mode locking of a Ti : sapphire laser," Appl. Phys. Lett., vol. 56, pp. 814-815, 1990.

[4] D. E. Spence, P. N. Kean, and W. Sibbett, "Sub-100 fsec pulse generation from a self-modulated titanium-sapphire laser," in Conf. Dig. LEOS, Wash. DC: OSA, 1990, paper PDP10

15] L. F. Mollenauer, N. D. Vieira, and L. Szeto, "Mode locking by synchronous pumping using a gain medium with microsecond decay times," Opt. Lett., vol. 7, pp. 414-416, 1982.

[6] P. M. W. French, S. M. J. Kelly, and J. R. Taylor, "Mode-locking of a continuous wave titanium doped sapphire laser using a linear extemal cavity," Opt. Lett., vol. 15, pp. 378-380, 1990; J. Mark, L. Y. Liu, K. L. Hall, H. A. Haus, and E. P. Ippen, "Femtosecond pulse generation in a laser with a nonlinear external resonator," $O p t$. Lett., vol. 14, pp. 48-50, 1989.

17] V. Keller, W. H. Knox, and H. Roskos, "Cavity coupled resonant passive mode locked $\mathrm{Ti}: \mathrm{Al}_{2} \mathrm{O}_{3}$ laser," in Proc., 7th Int. Conf. Ultrafast Phenom., C. B. Harris, E. P. Ippen, G. A. Mouron, and A. H. Zewail, Eds. Berlin: Springer-Verlag, 1990, pp. 69-7I.
[8] J. T. Darrow and R. K. Jain, "Active mode locking of broad band continuous wave lasers," IEEE J. Quantum Electron., vol. 27, pp $1048-1060,1991$; and the references therein.

[9] M. T. Asaki, C. P. Huang, D. Garvey, J. Zhou, H. Nathel, C. Kapteyn and M. M. Murname, "11 femtosecond pulses from a mode locked Ti: sapphire laser," in Tech. Dig., OSA Meet., 1992, Albuquerque, NM, paper PD17.

[10] D. J. Kuizenga and A. E. Siegman, "FM and AM mode-locking of the homogeneous laser - Part I: Theory," IEEE J. Quantum Electron., vol. QE-6, pp. 694-708, 1970.

[11] J. Goodberlet, J. Wang, and J. G. Fujimoto, "Starting dynamics of additive-pulse mode locking in the $\mathrm{Ti}: \mathrm{Al}_{2} \mathrm{O}_{3}$ laser," Opt. Lett., vol. 15, pp. 1300-1302, 1990.

[12] Y. Ishida, N. Sarukura, and H. Nakano, in Conf. Dig., CLEO, Wash. DC: OSA 1991, paper JMB2.

[13] C.-L. Pan, J.-C. Kuo, K.-H. Wu, J.-M. Shieh, C.-D. Hwang, and C. S. Chang, "Pulse forming dynamics of a $\mathrm{CW}$ passively mode locked Ti: sapphire/DDI laser," in Tech. Dig., CLEO/QELS Meet. Anaheim, CA: OSA, 1992, paper CTu P2,; Opt. Lett., vol. 17, pp. 334-336, 1992.

[14] N. P. Barnes, "Mode-locking dynamics of homogeneously broadened lasers," J. Appl. Phys., vol. 45, pp. 1291-1297, 1974.

[15] K. P. J. Reddy, "Transient pulse evolution of active mode locking in an intracavity frequency-doubled laser," $J$. Appl. Phys., vol. 53, pp. $7076-7079,1982$.

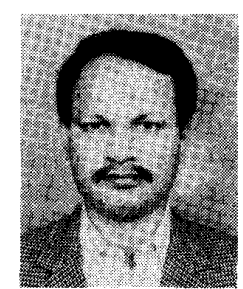

K. P. J. Reddy was born on January 9,1953 in Karnataka. India. He received the B.Sc. and M.Sc. degrees in physics from the University of Mysore, Karnataka, India, in 1973 and 1975, respectively, and the $\mathrm{Ph} . \mathrm{D}$. degree in engineering from Birla Institute of Technology, Mesra, Ranchi, India, in 1981.

He joined the Indian Institute of Science, Bangalore, as a Project Assistant in 1981 and became Senior Scientific Officer with the Department of Aerospace Engineering in 1984. Currently, he is working as an Assistant Professor with the same department. From February to August 1992, he worked as a visiting Assistant Professor with the Electrical Engineering Department of the University of Texas at Dallas and as a Visiting Professor at the Center for High Technology Materials, University of New Mexico, Albuquerque, from August to February 1993, under the Indo-US Exchange Program. His areas of interest are theoretical and experimental analysis of mode locking and frequency doubling in lasers, gasdynamic lasers, and aerodynamics. During his stay in the U.S., he worked on mode locking and frequency doubling in $\mathrm{Ti}$ : sapphire lasers and mode locking and gain switching of semiconductor lasers. Recently, he built a shock-tube-driven high-power carbon dioxide laser. His other areas of interest include theoretical analysis of application of organic dye molecules and bio-molecules for spatial light modulators. He has also authored or co-authored over 60 technical papers in these areas. He is a consultant for the Implementation of Laser courses in Polytechnics, India.

Dr. Reddy is a member of the Optical Society of America, the American Institute of Aeronautics and Astronautics, and the Aeronautical Society of India. He is listed in India's Who's Who and Reference Asia: Men and Women of Achievement in Asia.

Jim A. Tatum received the B.S. degree in physics from Austin College, and the M.S. degree in electrical engineering from The University of Texas at Dallas.

$\mathrm{He}$ is currently pursuing the Ph.D. degree at the Laser Electronics Laboratory at UTD. Specifically, he is studying diode lasers and their applications 